\title{
Strict Detailed Balance is Unnecessary in Monte Carlo Simulation
}

\author{
Vasilios I. Manousiouthakis and Michael W. Deem \\ Chemical Engineering Department \\ University of California \\ Los Angeles, CA 90095-1592
}

\begin{abstract}
Detailed balance is an overly strict condition to ensure a valid Monte Carlo simulation. We show that, under fairly general assumptions, a Monte Carlo simulation need satisfy only the weaker balance condition. Not only does our proof show that sequential updating schemes are correct, but also it establishes the correctness of a whole class of new methods that simply leave the Boltzmann distribution invariant.
\end{abstract}

To appear in J. Chem. Phys. 
The theoretical basis for Monte Carlo simulation is Markov chain theory. In a Monte Carlo simulation, the configuration of a system of interest is updated by a series of moves, and the moves are chosen so that the updating process forms a Markov chain. Let us consider, without loss of generality, that the system is in the canonical ensemble. We then desire that the configurations generated by the Markov chain sample the Boltzmann distribution, after an initial transient, "equilibration" period. We would like, in other words, that the limiting distribution of the Markov chain exists, is unique, and is the Boltzmann distribution. This result is assured if the Markov chain is regular and satisfies the detailed balance condition. 1 The proof of this result uses the Perron-Frobenius theorem and the fact that a matrix obeying detailed balance has a complete set of eigenvectors.

There are many Monte Carlo methods in wide use, however, that do not satisfy detailed balance. The classic example is the sequential updating method. I $^{\text {In }}$ such a method, the reverse move of sequentially updating in the reverse order never occurs, and so the method cannot satisfy detailed balance. The correctness of these simulations cannot be justified with the standard proof, which relies on the detailed balance condition. In fact, there appears to be no proof available in the literature that would assure us that these methods are correct. Yet, they are in wide use. Many specific examples can be given. One recent example is sequential updating of spins in the Ising model. l $_{\text {Another is sequential updating }}$ of surface patches in membrane simulations. 6 Of interest to the chemistry and chemical engineering community is the original implementation of the Gibbs ensemble, where particle displacements, volume changes, and particle exchanges were carried out in a fixed order. All implementations to date of parallel tempering, a powerful method for sampling "glassy" systems, perform a fixed sequence of particle moves and system swaps 3 . 12 Finally, several hybrid methods that combine Monte Carlo and molecular dynamics in a sequential fashion have been introduced. 13 variety of more general methods have been introduced that are guaranteed only to leave the Boltzmann distribution invariant, not to converge to it 14 Given the lack of a rigorous proof of correctness of these methods and the general belief in the importance of detailed balance, these methods have been criticized by various authors.

In this short communication, we show that detailed balance is too strict a condition. In particular, we show that the weaker balance condition is sufficient and necessary. The balance condition simply requires that the Markov chain leave the Boltzmann distribution invariant. To be precise, we show that a set of Monte Carlo moves is valid if they 1) lead to Markov sampling, 2) lead to regular sampling, and 3) satisfy the balance condition.

The organization of our paper is as follows. We first formulate in matrix notation the master equation that governs the Markov process. Next, we state precisely our theorem. 
We then prove the theorem, first in a heuristic way and then in a rigorous, formal fashion. We conclude with a discussion of the implications of this theorem.

A Monte Carlo simulation can be understood as an implementation of a discrete-time master equation. The state of the system at $n$ Monte Carlo steps (MCS) is related to that at $n-1$ MCS by a transition matrix:

$$
\mathbf{x}_{n}=A \mathbf{x}_{n-1}
$$

where the probability that the system is in state $i$ at $n$ MCS is given by the i-th component of the vector vector $\mathbf{x}_{n}$, and $A$ is the transition matrix. In this formulation, the sequence of steps forms a Markov chain. For a method that satisfies detailed balance, each step can be one of the many possible moves, chosen at random. For a sequential updating procedure, each step will be an entire sweep through a sequential series of moves.

The transition matrix satisfies certain obvious properties. The first is that the matrix is non-negative:

$$
A_{i j} \geq 0 \quad \forall i, \quad \forall j
$$

which ensures that the probabilities remain positive. The second obvious condition is that the transpose of the matrix is stochastic:

$$
\sum_{i} A_{i j}=1 \quad \forall j
$$

which ensures that the transition matrix conserves total probability. It is well known that the set of Monte Carlo moves must lead to ergodic sampling, otherwise the system will not sample all of phase space. In matrix notation, this is expressed as $\left[A^{m}\right]_{i j}>0$ for all $i$ and $j$, for some $m$, which may depend on $i$ and $j$. We will need the slightly stronger condition that the set of moves leads to regular sampling. This condition is expressed as

$$
\left[A^{m}\right]_{i j}>0 \quad \text { for all } i, j \text { for some fixed } m \text {. }
$$

Note that a set of moves that leads to ergodic sampling can be converted into a set of moves that leads to regular sampling with the addition of some null moves:

$$
A^{\prime}=(1-\alpha) A+\alpha I, \quad(0<\alpha<1)
$$

where $I$ is the identity matrix. Finally, if the desired distribution, i.e. the Boltzmann distribution, is denoted by $\mathbf{x}^{*}$, the balance condition is simply that

$$
A \mathrm{x}^{*}=\mathrm{x}^{*}
$$


We note that the detailed balance condition is expressed in matrix notation as $A_{i j} x_{j}^{*}=A_{j i} x_{i}^{*}$. We further note that if matrix $A$ satisfies the balance condition, then the matrix $A^{\prime}$ in Eq. (5) also satisfies the same balance condition.

Our theorem is that a set of Markov moves satisfying conditions (2-1) always converges to a limiting distribution, which is unique. It follows that if by some means we know that the moves satisfy the balance condition (6), then the simulation will eventually converge to sampling the desired distribution $\mathrm{x}^{*}$.

We first present a simple proof of this theorem. The Perron theorem implies that the matrix $A^{m}$ has a single, unique maximum eigenvalue, $\lambda$, with an associated eigenvector, $\mathbf{x}^{*}$, with non-negative entries (Theorems 1.4.4 and 1.8.1 of Ref. 15). By taking a sum over components in the equation $A^{m} \mathbf{x}^{*}=\lambda \mathbf{x}^{*}$, we conclude that $\lambda=1$. The limiting distribution, if it exists, is given by $\lim _{n \rightarrow \infty} A^{m n} \mathbf{x}_{0}$, where $\mathbf{x}_{0}$ is the initial condition. Since the sampling is regular, this limit cannot depend on the initial condition, because essentially all initial conditions are sampled during the initial equilibration period. By choosing an initial condition of $\mathbf{x}_{0}=\mathbf{x}^{*}$, we conclude that the limiting distribution of $A^{m}$ is $\mathbf{x}^{*}$. Since the initial condition is irrelevant, we can equally well consider limits such as $\lim _{n \rightarrow \infty} A^{m n}\left[A^{k} \mathbf{x}_{0}\right]$, which simply correspond to different choices for initial conditions. But these limits all must be $\mathbf{x}^{*}$ for any $k$ by the same argument. That all of these limits are equal implies that

$$
\lim _{n \rightarrow \infty} A^{n} \mathbf{x}_{0}=\mathbf{x}^{*},
$$

where the $m$ has been eliminated, and the theorem is proved.

We now present an alternative and more formal proof of the theorem. From conditions (2) and (3) and Lemma 3.1.1 of Ref. 15 we conclude that the spectral radius of $A$ is unity. From conditions (2)-(1) and the Perron-Frobenius theorem (Theorem 1.4.4 of Ref. 15) we conclude that 1 is an eigenvalue of $A$ and that the components of the corresponding eigenvector are non-negative. We denote this eigenvector by $\mathbf{w}^{(1)}$. From condition (四) we can, in fact, reach the stronger conclusion that all other eigenvalues of $A$ have a modulus strictly less than unity (Theorem 1.8.1 of Ref. 15)). Page 40 of Ref. 15 then states that

$$
\lim _{n \rightarrow \infty} A^{n}=\lim _{\lambda \rightarrow 1^{+}}\left[(\lambda-1)(\lambda I-A)^{-1}\right] .
$$

We now proceed to evaluate the limit in Eq. (8). We first decompose the $l \times l$ matrix $A$ into its Jordan normal form: $\mathbb{1}^{-1} A=W J W^{-1}$, where

$$
J=\left[\begin{array}{ccccc}
\lambda_{1} & b_{1} & & & 0 \\
& \lambda_{2} & b_{2} & & \\
& & \ddots & \ddots & \\
& & & \lambda_{l-1} & b_{l-1} \\
0 & & & & \lambda_{l}
\end{array}\right] .
$$


Here $\lambda_{i}$ are the eigenvalues of $A$, and $b_{i}$ are either 0 or 1 depending on the degrees of the elementary divisors. We find that

$$
\begin{aligned}
\lim _{\lambda \rightarrow 1^{+}}\left[(\lambda-1)(\lambda I-A)^{-1}\right] & =\lim _{\lambda \rightarrow 1^{+}}\left[W(\lambda-1)(\lambda I-J)^{-1} W^{-1}\right] \\
& =W\left[\lim _{\lambda \rightarrow 1^{+}}\left[(\lambda-1)(\lambda I-J)^{-1}\right]\right] W^{-1}
\end{aligned}
$$

Carrying out the inversion, we find

$$
(\lambda I-J)^{-1}=\left[\begin{array}{cccccc}
\frac{1}{\lambda-1} & \frac{b_{1}}{(\lambda-1)\left(\lambda-\lambda_{2}\right)} & \frac{b_{1} b_{2}}{(\lambda-1)\left(\lambda-\lambda_{2}\right)\left(\lambda-\lambda_{3}\right)} & \frac{b_{2}}{(\lambda-1)\left(\lambda-\lambda_{2}\right)\left(\lambda-b_{3}\right)\left(\lambda-\lambda_{4}\right)} & \cdots \\
0 & \frac{1}{\lambda-\lambda_{2}} & \frac{b_{2} b_{3}}{\left(\lambda-\lambda_{2}\right)\left(\lambda-\lambda_{3}\right)} & \frac{1}{\left(\lambda-\lambda_{2}\right)\left(\lambda-\lambda_{3}\right)\left(\lambda-\lambda_{4}\right)} & \cdots \\
0 & 0 & \frac{b_{3}}{\lambda-\lambda_{3}} & \frac{b_{3}}{\left(\lambda-\lambda_{3}\right)\left(\lambda-\lambda_{4}\right)} & \cdots \\
0 & 0 & 0 & \frac{1}{\lambda-\lambda_{4}} & \cdots \\
\vdots & \vdots & \vdots & \vdots & \ddots & \\
0 & 0 & 0 & 0 & \frac{1}{\lambda-\lambda_{l}}
\end{array}\right] .
$$

Now since $\lambda_{1}=1$ is the only eigenvalue of unit modulus, the associated elementary divisor has degree 1 , and $b_{1}=0$. We then see that

$$
\lim _{\lambda \rightarrow 1^{+}}\left[(\lambda-1)(\lambda I-J)^{-1}\right]=\left[\begin{array}{ccc}
1 & 0 & \cdots \\
0 & 0 & \cdots \\
\vdots & \vdots & \ddots
\end{array}\right]=\mathbf{e e}^{\top},
$$

where $e_{1}=1$, and $e_{i}=0$ otherwise. We denote the columns of $W$ by $\mathbf{w}^{(i)}$. Since $W$ is by definition a non-singular matrix, 16 the vectors $\mathbf{w}^{(i)}$ form a complete basis, and we can decompose the initial condition as $\mathbf{x}_{0}=\sum_{i=1}^{l} a_{i} \mathbf{w}^{(i)} \equiv W \mathbf{a}$. From Eqs. (8), (10), and (12), we have

$$
\begin{aligned}
\lim _{n \rightarrow \infty} A^{n} \mathbf{x}_{0} & =\lim _{\lambda \rightarrow 1^{+}}\left[(\lambda-1)(\lambda I-A)^{-1}\right] \mathbf{x}_{0} \\
& =W \mathbf{e e}^{\top} W^{-1} W \mathbf{a} \\
& =W \mathbf{e e}^{\top} \mathbf{a} \\
& =a_{1} \mathbf{w}^{(1)} \\
& =\mathbf{w}^{(1)}
\end{aligned}
$$

where in the last step we have used Eq. (3) and the fact that both $\mathbf{x}_{0}$ and $\mathbf{w}^{(1)}$ are normalized probability distributions. This completes the rigorous proof of the theorem.

The implications of our theorem are significant. First, the sequential updating approach, widely practiced and nearly as widely criticized, is correct. All that is required is that the sweep leave the Boltzmann distribution invariant, in addition to leading to regular sampling. Indeed, there are numerical reports that the sequential updating approach leads not only to 
correct sampling but also to more efficient sampling in a variety of contexts, ranging from image processing to membrane physics. 1 There are some simple cases, however, for which random Metropolis updating is ergodic and regular, but sequential Metropolis updating is not. One is the Ising model at infinite temperature, where the configurations produced by sequential updating simply oscillate between the initial configuration and the configuration with all spins flipped. Another example is the $2 \times 2$ Ising model at any temperature. If the spins are updated in the order $\left[\begin{array}{ll}1 & 2 \\ 3 & 4\end{array}\right]$, then the initial configurations $\left[\begin{array}{ll}+ & - \\ + & +\end{array}\right]$ and $\left[\begin{array}{l}+ \\ - \\ +\end{array}\right]$ lead to sequences that also oscillate between spin-flipped configurations. In these examples, our theorem does not guarantee convergence to the Boltzmann distribution, because assumption (田) is violated. If, however, the individual spin-flip moves within the sweep were performed with a probability $1-\alpha$, as in Eq. (5), then convergence to the Boltzmann distribution would occur in both of these examples.

Note that our theorem requires the sampling to be regular, not simply ergodic. Indeed, a method that is ergodic may not converge to the invariant distribution, even if it satisfies detailed balance. A simple example is provided by a two-state system. The transition matrix $\left[\begin{array}{ll}0 & 1 \\ 1 & 0\end{array}\right]$ leads to ergodic, but not regular, sampling. This transition matrix satisfies detailed balance with the invariant distribution $x_{1}^{*}=x_{2}^{*}=1 / 2$. The $\operatorname{limit}_{n \rightarrow \infty} A^{n} x_{0}$ does not exist for a general initial condition, however, because $A^{n} x_{0}$ simply alternates between $x_{0}$ and the swapped state for even and odd $n$.

There has been concern that perhaps one should not use the data obtained from within the sweep of a sequential move, or that perhaps the statistics collected would depend on how the sweep is defined. In fact, we can show that all of the data can be used, and the results do not depend on how the sweep is defined. For simplicity, let us consider a sweep composed of $N$ steps: $A=\prod_{k=1}^{N} B^{(k)}$. Our general theorem says that the data collected via the procedure $\mathbf{x}_{n}=A^{n} \mathbf{x}_{0}$ will sample the Boltzmann distribution. Moreover, after the initial equilibration period, the initial condition $\mathbf{x}_{0}$ will not affect the results. We can, therefore, equally well consider the exact same sequence of moves but collect data at a shifted period within the sweep: $\mathbf{x}_{n}=\left[B^{(N)} \prod_{k=1}^{N-1} B^{(k)}\right]^{n}\left[B^{(N)} \mathbf{x}_{0}\right]$. This approach, which uses the data within the sweep, is also susceptible to our general theorem. We can generalize this argument to any arbitrary shifting of the data collection period. By our theorem, all of the data so collected will be sampling from the Boltzmann distribution once the equilibration regime has passed. The data from within the sweep, therefore, can be used, and the average statistics will not depend on which data are used.

We note that a simple way to ensure that a sequential sweep satisfy the balance condition is to require that each of the moves within the sweep satisfy local detailed balance: $B_{i j}^{(k)} x_{j}^{*}=$ 
$B_{j i}^{(k)} x_{i}^{*}$. Indeed, the local detailed balance condition is satisfied in all of the sequential Monte Carlo methods previously cited. By using this condition, one can show that the balance condition, Eq. (6), is satisfied:

$$
\begin{aligned}
{\left[A \mathbf{x}^{*}\right]_{i} } & =\left[\prod_{k=1}^{N} B^{(k)} \mathbf{x}^{*}\right]_{i} \\
& =\sum_{i_{1}, i_{2}, \ldots, i_{N}} B_{i i_{1}}^{(1)} B_{i_{1} i_{2}}^{(2)} \cdots B_{i_{N-2} i_{N-1}}^{(N-1)} B_{i_{N-1} i_{N}}^{(N)} x_{i_{N}}^{*} \\
& =\sum_{i_{1}, i_{2}, \ldots, i_{N}} B_{i i_{1}}^{(1)} B_{i_{1} i_{2}}^{(2)} \cdots B_{i_{N-2} i_{N-1}}^{(N-1)} x_{i_{N-1}}^{*} B_{i_{N} i_{N-1}}^{(N)} \\
& =\sum_{i_{1}, i_{2}, \ldots, i_{N-1}} B_{i i_{1}}^{(1)} B_{i_{1} i_{2}}^{(2)} \cdots B_{i_{N-2} i_{N-1}}^{(N-1)} x_{i_{N-1}}^{*} \\
& \vdots \\
& =x_{i}^{*}
\end{aligned}
$$

Here we have used local detailed balance condition to achieve the third line and the fact that the transpose of each $B^{(k)}$ is stochastic to achieve the fourth line. The final line is achieved by induction.

More generally, our result implies that any Monte Carlo method that leaves the Boltzmann distribution invariant is correct as long as it leads to regular, Markov sampling. The type-R and type-M transitions within the dynamic weighting method of Wong and Liang,

for example, are known only to leave the Boltzmann distribution invariant 14 Although the method led to efficient and apparently accurate sampling, the formal correctness of this method has not been shown. Our general theorem formally establishes the validity of this and other such methods.

In summary, we have clarified a long-standing and controversial issue in Monte Carlo simulations by establishing the balance condition as a necessary and sufficient fundamental requirement. This condition is substantially weaker than the detailed balance condition. Although our proof of convergence demands that the set of Monte Carlo moves lead to regular sampling, this is the case for almost all sets of moves that lead to ergodic sampling. We have shown that the local detailed balance condition is one means of constructing a sequential Monte Carlo scheme satisfying the balance condition.

\section{ACKNOWLEDGMENT}

This research was supported by the National Science Foundation. It is a pleasure to acknowledge stimulating discussions with Daan Frenkel, Giorgio Parisi, and Alan D. Sokal. 


\section{REFERENCES}

${ }^{1}$ G. Parisi, Statistical Field Theory, Vol. 66 of Frontiers in Physics (Addison-Wesley Pub. Co., Redwood City, CA, 1988), p. 343.

${ }^{2}$ D. Frenkel and B. Smit, Understanding Molecular Simulations (Academic Press, San Diego, 1996), p. 50.

${ }^{3}$ E. Marinari, G. Parisi, and J. Ruiz-Lorenzo, in Spin Glasses and Random Fields, Vol. 12 of Directions in Condensed Matter Physics, edited by A. Young (World Scientific, Singapore, 1998).

${ }^{4}$ G. Thorleifsson and M. Falcioni, Computer Physics Communications 109, 161 (1998).

${ }^{5}$ A. Z. Panagiotopoulos, Mol. Phys. 61, 813 (1987).

${ }^{6}$ C. J. Geyer, in Computing Science and Statistics: Proceedings of the 23rd Symposium on the Interface (American Statistical Association, 1991), p. 156.

${ }^{7}$ C. J. Geyer and E. A. Thompson, J. Am. Stat. Assn. 90, 909 (1995).

${ }^{8}$ K. Hukushima and K. Nemoto, J. Phys. Soc. Japan 65, 1604 (1996).

${ }^{9}$ M. C. Tesi, E. J. Janse van Rensburg, E. Orlandini, and S. G. Whittington, J. Stat. Phys. 82,155 (1996).

${ }^{10}$ M. C. Tesi, E. J. Janse van Rensburg, E. Orlandini, and S. G. Whittington, J. Phys. A 29, 2451 (1996).

${ }^{11}$ G. Boyd, Nucl. Phys. B Supplement 60A, 341 (1998).

12 U. H. E. Hansmann, Chem. Phys. Lett. 281, 140 (1997).

${ }^{13}$ P. G. Bolhuis, C. Dellago, and D. Chandler, Faraday Discuss. Chem. Soc. 110, 421 (1998).

${ }^{14}$ W. H. Wong and F. Liang, Proc. Natl. Acad. Sci. USA 94, 14220 (1997).

${ }^{15}$ R. B. Bapat and T. E. S. Raghavan, Nonnegative Matrices and Applications (Cambridge University Press, New York, 1997).

${ }^{16}$ F. R. Gantmacher, Matrix Theory (Chelsea, New York, 1959), Vol. 1, p. 152. 